\title{
Density Functional Studies on Dicobalt Octacarbonyl Mediated Urea Formation from Primary Amine
}

\author{
Fung-E Hong*, Yu-Chang Chang \\ Department of Chemistry, National Chung-Hsing University, \\ Taichung 40227, Taiwan
}

\section{Supporting Information:}

I. Optimized Geometries and energies of the Insertion-Addition pathway at B3LYP/631LAN level of theory (LANL2DZ for Co and 6-31G(d) basis set for the other atoms)

II. Optimized Geometries and energies of the Addition-Insertion pathway at B3LYP/631LAN level of theory (LANL2DZ for Co and 6-31G(d) basis set for the other atoms) 
I. Optimized Geometries and energies of the Insertion-Addition pathway:

\section{Cartesian coordinates of CP1 (B3LYP-631LAN)}

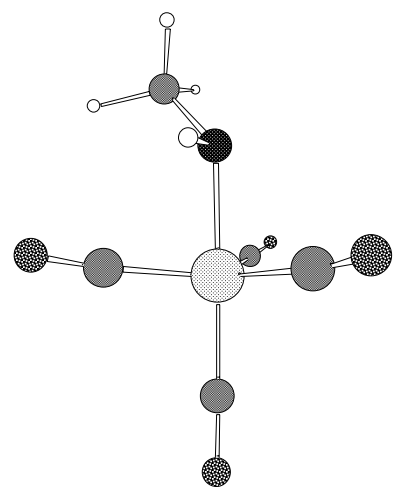

SCF Done: $\quad E(R B+H F-L Y P)=-693.658189829 \quad$ A.U.

\begin{tabular}{|c|c|c|c|c|c|}
\hline \multirow{2}{*}{$\begin{array}{l}\text { Center } \\
\text { Number }\end{array}$} & \multirow{2}{*}{$\begin{array}{l}\text { Atomic } \\
\text { Number }\end{array}$} & \multirow{2}{*}{$\begin{array}{l}\text { Atomic } \\
\text { Type }\end{array}$} & \multicolumn{3}{|c|}{ Coordinates (Angstroms) } \\
\hline & & & $\mathrm{X}$ & $\mathrm{Y}$ & Z \\
\hline 1 & 27 & 0 & 0.209896 & 0.029613 & -0.038835 \\
\hline 2 & 7 & 0 & -1.602335 & -0.512735 & -0.683295 \\
\hline 3 & 1 & 0 & -1.735529 & -0.026616 & -1.570569 \\
\hline 4 & 6 & 0 & -2.759339 & -0.215606 & 0.160217 \\
\hline 5 & 1 & 0 & -3.667787 & -0.529394 & -0.374844 \\
\hline 6 & 1 & 0 & -2.895436 & 0.842723 & 0.444749 \\
\hline 7 & 1 & 0 & -2.711821 & -0.801732 & 1.084108 \\
\hline 8 & 6 & 0 & 1.883702 & 0.585641 & 0.389562 \\
\hline 9 & 8 & 0 & 2.935870 & 0.954800 & 0.649428 \\
\hline 10 & 6 & 0 & 0.732328 & -1.174883 & -1.338439 \\
\hline 11 & 8 & 0 & 0.928146 & -1.953814 & -2.153505 \\
\hline 12 & 6 & 0 & -0.435976 & 1.680108 & -0.370188 \\
\hline 13 & 8 & 0 & -0.942797 & 2.688155 & -0.592636 \\
\hline 14 & 6 & 0 & -0.112111 & -0.799894 & 1.575611 \\
\hline
\end{tabular}




\section{Cartesian coordinates of TS1 (B3LYP-631LAN)}

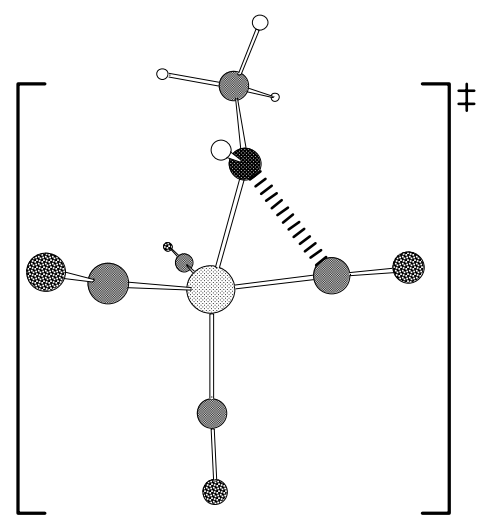

SCF Done: $\quad \mathrm{E}(\mathrm{RB}+\mathrm{HF}-\mathrm{LYP})=-693.654836546 \quad$ A.U.

\begin{tabular}{|c|c|c|c|c|c|}
\hline \multirow{2}{*}{$\begin{array}{l}\text { Center } \\
\text { Number }\end{array}$} & \multirow{2}{*}{$\begin{array}{l}\text { Atomic } \\
\text { Number }\end{array}$} & \multirow{2}{*}{$\begin{array}{l}\text { Atomic } \\
\text { Type }\end{array}$} & \multicolumn{3}{|c|}{ Coordinates (Angstroms) } \\
\hline & & & $\mathrm{X}$ & $\mathrm{Y}$ & $\mathrm{Z}$ \\
\hline 1 & 27 & 0 & -0.224506 & -0.002070 & -0.042210 \\
\hline 2 & 7 & 0 & 1.626308 & 0.076690 & -0.708308 \\
\hline 3 & 1 & 0 & 1.676123 & -0.304710 & -1.647350 \\
\hline 4 & 6 & 0 & 2.735523 & -0.347752 & 0.128313 \\
\hline 5 & 1 & 0 & 3.682259 & -0.193989 & -0.408462 \\
\hline 6 & 1 & 0 & 2.691865 & -1.404687 & 0.442634 \\
\hline 7 & 1 & 0 & 2.770911 & 0.268271 & 1.034028 \\
\hline 8 & 6 & 0 & -1.958764 & 0.419391 & 0.222360 \\
\hline 9 & 8 & 0 & -3.052907 & 0.725504 & 0.365858 \\
\hline 10 & 6 & 0 & -0.535613 & -1.458302 & -1.090949 \\
\hline 11 & 8 & 0 & -0.622392 & -2.374969 & -1.777314 \\
\hline 12 & 6 & 0 & 0.167609 & -0.470543 & 1.702361 \\
\hline 13 & 8 & 0 & 0.410747 & -0.761684 & 2.783816 \\
\hline 14 & 6 & 0 & 0.344632 & 1.647580 & -0.549147 \\
\hline 15 & 8 & 0 & 0.681555 & 2.712642 & -0.847442 \\
\hline
\end{tabular}




\section{Cartesian coordinates of CP2 (B3LYP-631LLAN)}

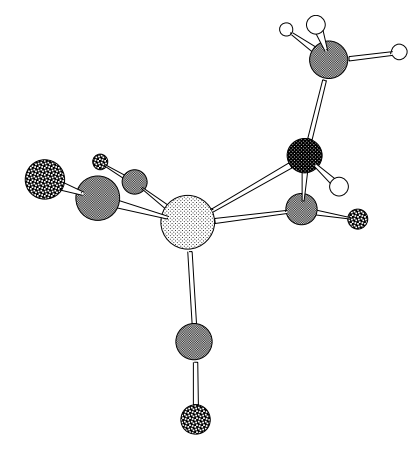

SCF Done: $\quad E(R B+H F-L Y P)=-693.674728043 \quad$ A.U.

\begin{tabular}{|c|c|c|c|c|c|}
\hline \multirow{2}{*}{$\begin{array}{l}\text { Center } \\
\text { Number }\end{array}$} & \multirow{2}{*}{$\begin{array}{l}\text { Atomic } \\
\text { Number }\end{array}$} & \multirow{2}{*}{$\begin{array}{l}\text { Atomic } \\
\text { Type }\end{array}$} & \multicolumn{3}{|c|}{ Coordinates (Angstroms) } \\
\hline & & & $\mathrm{X}$ & $\mathrm{Y}$ & Z \\
\hline 1 & 27 & 0 & -0.314289 & 0.010717 & -0.013841 \\
\hline 2 & 7 & 0 & 1.463432 & -0.425002 & -1.015198 \\
\hline 3 & 1 & 0 & 1.485018 & -0.107390 & -1.980957 \\
\hline 4 & 6 & 0 & 2.472670 & -1.457092 & -0.727268 \\
\hline 5 & 1 & 0 & 3.489861 & -1.071428 & -0.869056 \\
\hline 6 & 1 & 0 & 2.307499 & -2.316704 & -1.382010 \\
\hline 7 & 1 & 0 & 2.356233 & -1.782793 & 0.308390 \\
\hline 8 & 6 & 0 & -1.115725 & 1.492025 & -0.607404 \\
\hline 9 & 8 & 0 & -1.652650 & 2.468170 & -0.896800 \\
\hline 10 & 6 & 0 & -1.581474 & -1.228056 & -0.472936 \\
\hline 11 & 8 & 0 & -2.325666 & -2.037600 & -0.798540 \\
\hline 12 & 6 & 0 & -0.294289 & -0.236056 & 1.755927 \\
\hline 13 & 8 & 0 & -0.327537 & -0.313361 & 2.903460 \\
\hline 14 & 6 & 0 & 1.376370 & 0.694478 & -0.070445 \\
\hline 15 & 8 & 0 & 2.238084 & 1.429314 & 0.308942 \\
\hline
\end{tabular}

\section{Cartesian coordinates of TS2 (B3LYP-631LAN)}




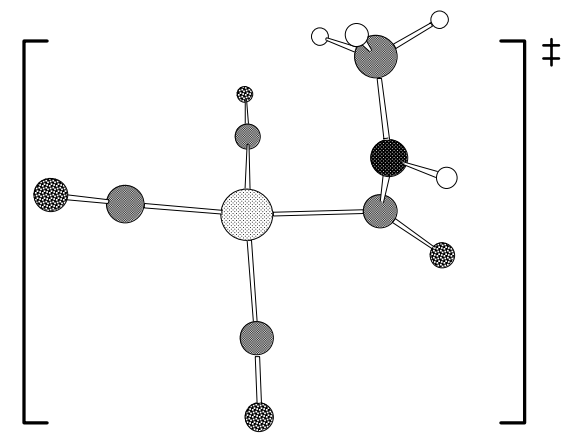

SCF Done: $\quad \mathrm{E}(\mathrm{RB}+\mathrm{HF}-\mathrm{LYP})=-693.664655199 \quad$ A.U.

\begin{tabular}{|c|c|c|c|c|c|}
\hline \multirow{2}{*}{$\begin{array}{l}\text { Center } \\
\text { Number }\end{array}$} & \multirow{2}{*}{$\begin{array}{l}\text { Atomic } \\
\text { Number }\end{array}$} & \multirow{2}{*}{$\begin{array}{l}\text { Atomic } \\
\text { Type }\end{array}$} & \multicolumn{3}{|c|}{ Coordinates (Angstroms) } \\
\hline & & & $\mathrm{X}$ & $\mathrm{Y}$ & $\mathrm{Z}$ \\
\hline 1 & 27 & 0 & -0.387709 & -0.010198 & -0.012518 \\
\hline 2 & 7 & 0 & 1.873774 & 0.060173 & -1.103770 \\
\hline 3 & 1 & 0 & 2.577046 & 0.674338 & -1.515474 \\
\hline 4 & 6 & 0 & 2.234518 & -1.359288 & -1.234165 \\
\hline 5 & 1 & 0 & 3.259474 & -1.552335 & -0.888982 \\
\hline 6 & 1 & 0 & 2.149039 & -1.667145 & -2.280352 \\
\hline 7 & 1 & 0 & 1.550853 & -1.970078 & -0.644093 \\
\hline 8 & 6 & 0 & -0.911879 & 1.708952 & 0.009632 \\
\hline 9 & 8 & 0 & -1.290163 & 2.780106 & 0.173632 \\
\hline 10 & 6 & 0 & -1.934865 & -0.746598 & -0.634429 \\
\hline 11 & 8 & 0 & -2.882466 & -1.224547 & -1.068207 \\
\hline 12 & 6 & 0 & -0.050617 & -1.163704 & 1.280447 \\
\hline 13 & 8 & 0 & 0.092866 & -1.822509 & 2.214581 \\
\hline 14 & 6 & 0 & 1.413556 & 0.634549 & 0.109855 \\
\hline 15 & 8 & 0 & 1.993644 & 1.507684 & 0.705648 \\
\hline
\end{tabular}

\section{Cartesian coordinates of CP3 (B3LYP-631LAN)}




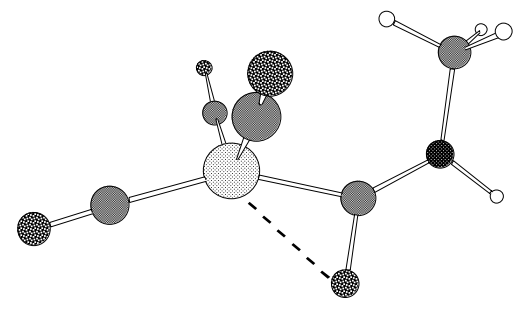

SCF Done: $\quad E(R B+H F-L Y P)=-693.691810136 \quad$ A.U.

\begin{tabular}{|c|c|c|c|c|c|}
\hline \multirow{2}{*}{$\begin{array}{l}\text { Center } \\
\text { Number }\end{array}$} & \multirow{2}{*}{$\begin{array}{l}\text { Atomic } \\
\text { Number }\end{array}$} & \multirow{2}{*}{$\begin{array}{l}\text { Atomic } \\
\text { Type }\end{array}$} & \multicolumn{3}{|c|}{ Coordinates (Angstroms) } \\
\hline & & & $\mathrm{X}$ & $\mathrm{Y}$ & $\mathrm{Z}$ \\
\hline 1 & 27 & 0 & -0.511278 & 0.003634 & 0.123655 \\
\hline 2 & 7 & 0 & 2.480365 & -0.020401 & 0.569911 \\
\hline 3 & 1 & 0 & 3.140899 & -0.048301 & 1.340875 \\
\hline 4 & 6 & 0 & 2.999542 & 0.024828 & -0.787670 \\
\hline 5 & 1 & 0 & 3.607079 & -0.861079 & -1.003170 \\
\hline 6 & 1 & 0 & 3.609259 & 0.921770 & -0.943159 \\
\hline 7 & 1 & 0 & 2.156922 & 0.049258 & -1.480432 \\
\hline 8 & 6 & 0 & -0.280408 & 1.636005 & -0.561819 \\
\hline 9 & 8 & 0 & -0.120824 & 2.628645 & -1.124916 \\
\hline 10 & 6 & 0 & -2.323179 & -0.005363 & 0.196700 \\
\hline 11 & 8 & 0 & -3.466425 & -0.012959 & 0.284456 \\
\hline 12 & 6 & 0 & -0.276715 & -1.573588 & -0.666044 \\
\hline 13 & 8 & 0 & -0.124825 & -2.594981 & -1.177446 \\
\hline 14 & 6 & 0 & 1.185504 & -0.026913 & 0.905220 \\
\hline 15 & 8 & 0 & 0.724491 & -0.064052 & 2.047845 \\
\hline
\end{tabular}

\section{Cartesian coordinates of CP4 (B3LYP-631LAN)}




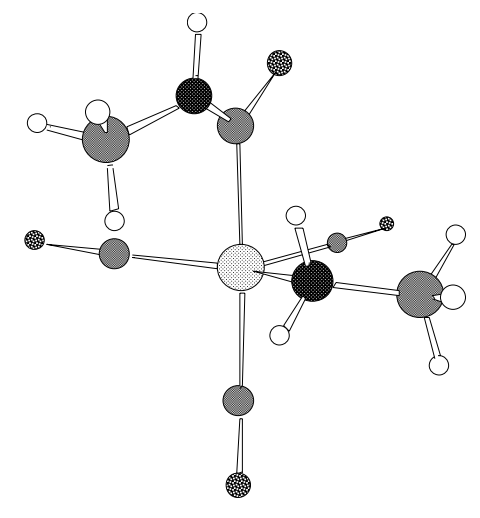

SCF Done: $\quad \mathrm{E}(\mathrm{RB}+\mathrm{HF}-\mathrm{LYP})=-789.551812392 \quad$ A.U.

\begin{tabular}{|c|c|c|c|c|c|}
\hline \multirow{2}{*}{$\begin{array}{l}\text { Center } \\
\text { Number }\end{array}$} & \multirow{2}{*}{$\begin{array}{l}\text { Atomic } \\
\text { Number }\end{array}$} & \multirow{2}{*}{$\begin{array}{l}\text { Atomic } \\
\text { Type }\end{array}$} & \multicolumn{3}{|c|}{ Coordinates (Angstroms) } \\
\hline & & & $\mathrm{X}$ & $\mathrm{Y}$ & $\mathrm{Z}$ \\
\hline 1 & 27 & 0 & 0.349546 & 0.257998 & 0.046814 \\
\hline 2 & 7 & 0 & -2.384416 & -0.721520 & -0.247582 \\
\hline 3 & 1 & 0 & -3.178132 & -1.186110 & 0.189882 \\
\hline 4 & 6 & 0 & -2.750733 & 0.244518 & -1.279580 \\
\hline 5 & 1 & 0 & -3.203032 & 1.158912 & -0.871942 \\
\hline 6 & 1 & 0 & -3.468900 & -0.224129 & -1.959978 \\
\hline 7 & 1 & 0 & -1.870159 & 0.536081 & -1.856005 \\
\hline 8 & 6 & 0 & 1.193733 & -0.450012 & 1.514090 \\
\hline 9 & 8 & 0 & 1.687940 & -0.769493 & 2.499353 \\
\hline 10 & 6 & 0 & 1.822844 & 1.026004 & -0.698361 \\
\hline 11 & 8 & 0 & 2.741081 & 1.503782 & -1.199621 \\
\hline 12 & 6 & 0 & -0.512468 & 1.778418 & 0.238450 \\
\hline 13 & 8 & 0 & -1.057707 & 2.777793 & 0.445141 \\
\hline 14 & 6 & 0 & -1.347260 & -0.577187 & 0.684320 \\
\hline 15 & 8 & 0 & -1.464133 & -1.128781 & 1.766904 \\
\hline 16 & 7 & 0 & 0.290652 & -1.234809 & -1.480229 \\
\hline 17 & 1 & 0 & -0.679971 & -1.551515 & -1.453426 \\
\hline 18 & 1 & 0 & 0.436105 & -0.814192 & -2.396374 \\
\hline 19 & 6 & 0 & 1.198004 & -2.391382 & -1.315435 \\
\hline 20 & 1 & 0 & 2.233628 & -2.042600 & -1.317161 \\
\hline 21 & 1 & 0 & 1.071983 & -3.138382 & -2.109917 \\
\hline 22 & 1 & 0 & 0.994916 & -2.858275 & -0.349484 \\
\hline
\end{tabular}




\section{Cartesian coordinates of TS3 (B3LYP-631LAN)}

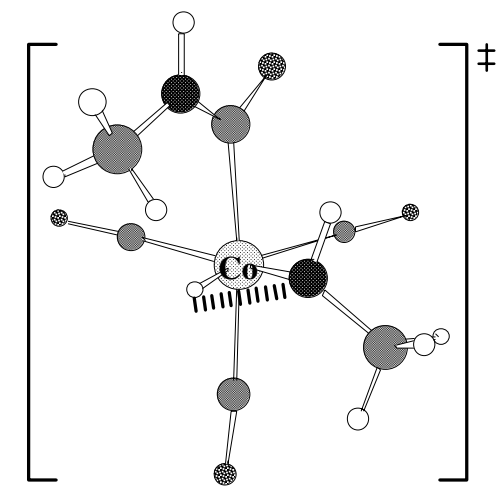

SCF Done: $\quad \mathrm{E}(\mathrm{RB}+\mathrm{HF}-\mathrm{LYP})=-789.466068625 \quad$ A.U.

\begin{tabular}{cccccc} 
Center & Atomic & Atomic & \multicolumn{3}{c}{ Coordinates (Angstroms) } \\
Number & Number & Type & X & Y & $Z$ \\
\hline 1 & 27 & 0 & 0.291423 & 0.218959 & -0.050858 \\
2 & 7 & 0 & -2.520393 & -0.677720 & -0.231920 \\
3 & 1 & 0 & -3.367325 & -0.961103 & 0.250481 \\
4 & 6 & 0 & -2.584463 & -0.540335 & -1.680534 \\
5 & 1 & 0 & -2.531623 & 0.506975 & -2.001374 \\
6 & 1 & 0 & -3.543434 & -0.947587 & -2.011525 \\
7 & 1 & 0 & -1.783779 & -1.106339 & -2.164356 \\
8 & 6 & 0 & 0.990061 & -0.115344 & 1.710409 \\
9 & 8 & 0 & 1.409642 & -0.419390 & 2.725600 \\
10 & 6 & 0 & 1.872262 & 0.801912 & -0.855505 \\
11 & 8 & 0 & 2.797053 & 1.226101 & -1.378344 \\
12 & 6 & 0 & -0.472098 & 1.846889 & 0.001995 \\
13 & 8 & 0 & -1.007123 & 2.861296 & -0.035374 \\
14 & 6 & 0 & -1.509620 & -0.351495 & 0.624214 \\
15 & 8 & 0 & -1.663186 & -0.452435 & 1.835262 \\
16 & 7 & 0 & 0.404058 & -1.605301 & -0.843589 \\
17 & 1 & 0 & -0.287706 & -2.160899 & -0.351376 \\
18 & 1 & 0 & -0.104118 & -0.167640 & -1.417572
\end{tabular}




$\begin{array}{rrrrrr}19 & 6 & 0 & 1.711839 & -2.236181 & -0.814474 \\ 20 & 1 & 0 & 2.396741 & -1.710855 & -1.492007 \\ 21 & 1 & 0 & 1.629508 & -3.269423 & -1.182468 \\ 22 & 1 & 0 & 2.198696 & -2.271130 & 0.178166\end{array}$

\section{Cartesian coordinates of CP5 (B3LYP-631LAN)}

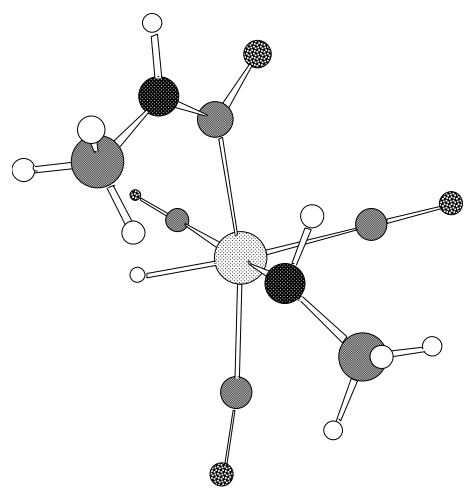

SCF Done: $\quad E(R B+H F-L Y P)=-789.480492320 \quad$ A.U.

\begin{tabular}{cccccc} 
Center & Atomic & Atomic & \multicolumn{3}{c}{ Coordinates (Angstroms) } \\
Number & Number & Type & $\mathrm{X}$ & $\mathrm{Y}$ & $\mathrm{Z}$ \\
\hline 1 & 27 & 0 & 0.257640 & 0.258638 & -0.142650 \\
2 & 7 & 0 & -2.439051 & -0.916849 & -0.309647 \\
3 & 1 & 0 & -3.382192 & -0.959199 & 0.065568 \\
4 & 6 & 0 & -2.189116 & -1.590235 & -1.577311 \\
5 & 1 & 0 & -2.460675 & -0.963441 & -2.437177 \\
6 & 1 & 0 & -2.797177 & -2.500105 & -1.608024 \\
7 & 1 & 0 & -1.135238 & -1.869608 & -1.629055 \\
8 & 6 & 0 & 0.712211 & 0.267669 & 1.675471 \\
9 & 8 & 0 & 0.975846 & 0.160290 & 2.779754 \\
10 & 6 & 0 & 1.908388 & 0.448479 & -1.012581 \\
11 & 8 & 0 & 2.834708 & 0.553115 & -1.672010 \\
12 & 6 & 0 & -0.294677 & 1.982325 & -0.389390 \\
13 & 8 & 0 & -0.680845 & 3.037339 & -0.592121 \\
14 & 6 & 0 & -1.647899 & -0.058822 & 0.383679 \\
& & & & & \\
& & & 9 & &
\end{tabular}




\begin{tabular}{rrrrrr}
15 & 8 & 0 & -2.057771 & 0.521680 & 1.382519 \\
16 & 7 & 0 & 0.499132 & -1.717484 & -0.062430 \\
17 & 1 & 0 & -0.183100 & -2.041449 & 0.623810 \\
18 & 1 & 0 & -0.229386 & 0.068783 & -1.528541 \\
19 & 6 & 0 & 1.801246 & -2.247725 & 0.337854 \\
20 & 1 & 0 & 2.551991 & -2.025043 & -0.430209 \\
21 & 1 & 0 & 1.730940 & -3.343860 & 0.403392 \\
22 & 1 & 0 & 2.211563 & -1.898500 & 1.304850 \\
\hline
\end{tabular}

\section{Cartesian coordinates of TS4a (B3LYP-631LAN)}

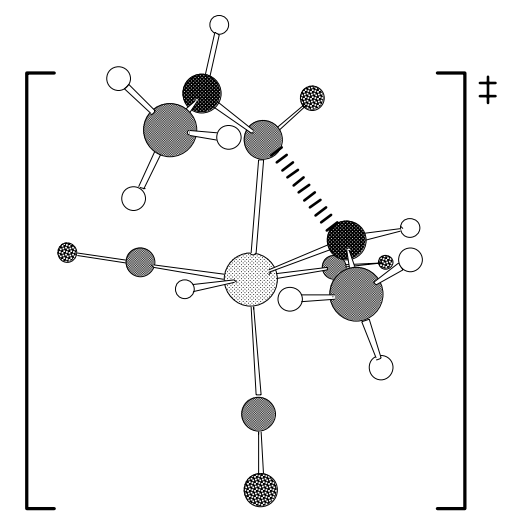

SCF Done: $\quad E(R B+H F-L Y P)=-789.472711201 \quad$ A.U.

\begin{tabular}{cccccc} 
Center & Atomic & Atomic & \multicolumn{3}{c}{ Coordinates (Angstroms) } \\
Number & Number & Type & $\mathrm{X}$ & $\mathrm{Y}$ & $\mathrm{Z}$ \\
\hline 1 & 27 & 0 & -0.451878 & -0.079248 & -0.166812 \\
2 & 7 & 0 & 2.507202 & -0.343830 & -0.179182 \\
3 & 1 & 0 & 3.292719 & -0.711939 & 0.350002 \\
4 & 6 & 0 & 2.829943 & 0.824076 & -0.978499 \\
5 & 1 & 0 & 2.058325 & 0.992207 & -1.733620 \\
6 & 1 & 0 & 3.773699 & 0.633669 & -1.497852 \\
7 & 1 & 0 & 2.931813 & 1.735564 & -0.374663 \\
8 & 6 & 0 & -1.258838 & -1.031925 & 1.231338 \\
9 & 8 & 0 & -1.783598 & -1.592177 & 2.073874 \\
10 & 6 & 0 & -1.946213 & 0.970950 & -0.701339
\end{tabular}




\begin{tabular}{|c|c|c|c|c|c|}
\hline 11 & 8 & 0 & -2.689460 & 1.756503 & -1.072764 \\
\hline 12 & 6 & 0 & -0.390542 & -1.379218 & -1.403988 \\
\hline 13 & 8 & 0 & -0.281335 & -2.161372 & -2.234090 \\
\hline 14 & 6 & 0 & 1.354748 & -0.535151 & 0.553280 \\
\hline 15 & 8 & 0 & 1.335621 & -1.271653 & 1.528785 \\
\hline 16 & 7 & 0 & 0.425142 & 1.206850 & 1.044090 \\
\hline 17 & 1 & 0 & 0.159928 & 1.011028 & 2.005315 \\
\hline 18 & 1 & 0 & 0.204949 & 0.674872 & -1.267193 \\
\hline 19 & 6 & 0 & 0.343608 & 2.627107 & 0.739952 \\
\hline 20 & 1 & 0 & 0.751750 & 2.816796 & -0.257741 \\
\hline 21 & 1 & 0 & 0.937654 & 3.203757 & 1.464571 \\
\hline 22 & 1 & 0 & -0.682580 & 3.037173 & 0.749853 \\
\hline
\end{tabular}

\section{Cartesian coordinates of CP6a (B3LYP-631LAN)}

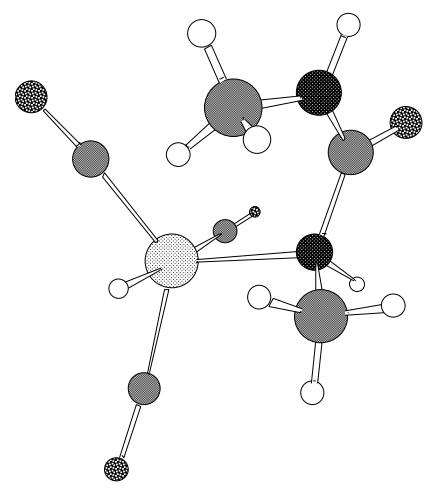

SCF Done: $\quad E(R B+H F-L Y P)=-789.553632117 \quad$ A.U.

\begin{tabular}{cccccc}
$\begin{array}{c}\text { Center } \\
\text { Number }\end{array}$ & Atomic & Atomic & \multicolumn{3}{c}{ Coordinates (Angstroms) } \\
\hline--------------- & Number & Type & X & Y & Z \\
1 & 27 & 0 & 0.904975 & 0.074233 & -0.232398 \\
2 & 7 & 0 & -2.818786 & 0.244576 & -0.021342 \\
3 & 1 & 0 & -3.499340 & 0.845506 & 0.423981 \\
4 & 6 & 0 & -2.738635 & 0.295930 & -1.476765 \\
5 & 1 & 0 & -1.698332 & 0.287611 & -1.806484 \\
6 & 1 & 0 & -3.190219 & 1.232129 & -1.816480
\end{tabular}




\begin{tabular}{|c|c|c|c|c|c|}
\hline 7 & 1 & 0 & -3.275737 & -0.534585 & -1.951403 \\
\hline 8 & 6 & 0 & 1.368103 & 0.664449 & 1.412765 \\
\hline 9 & 8 & 0 & 1.704066 & 1.064453 & 2.434227 \\
\hline 10 & 6 & 0 & 2.259107 & -1.031658 & -0.520107 \\
\hline 11 & 8 & 0 & 3.163263 & -1.689109 & -0.803853 \\
\hline 12 & 6 & 0 & 0.583475 & 1.652751 & -0.957451 \\
\hline 13 & 8 & 0 & 0.450129 & 2.657517 & -1.509090 \\
\hline 14 & 6 & 0 & -1.914215 & -0.220122 & 0.886795 \\
\hline 15 & 8 & 0 & -1.965191 & 0.063958 & 2.075192 \\
\hline 16 & 7 & 0 & -0.866685 & -1.089111 & 0.415832 \\
\hline 17 & 1 & 0 & -0.463310 & -1.441441 & 1.280855 \\
\hline 18 & 1 & 0 & 0.638717 & -0.327268 & -1.661038 \\
\hline 19 & 6 & 0 & -1.232956 & -2.244887 & -0.438893 \\
\hline 20 & 1 & 0 & -1.403053 & -1.930029 & -1.466761 \\
\hline 21 & 1 & 0 & -2.123880 & -2.761294 & -0.060822 \\
\hline 22 & 1 & 0 & -0.388288 & -2.936525 & -0.438391 \\
\hline
\end{tabular}

\section{Cartesian coordinates of TS4b (B3LYP-631LAN)}

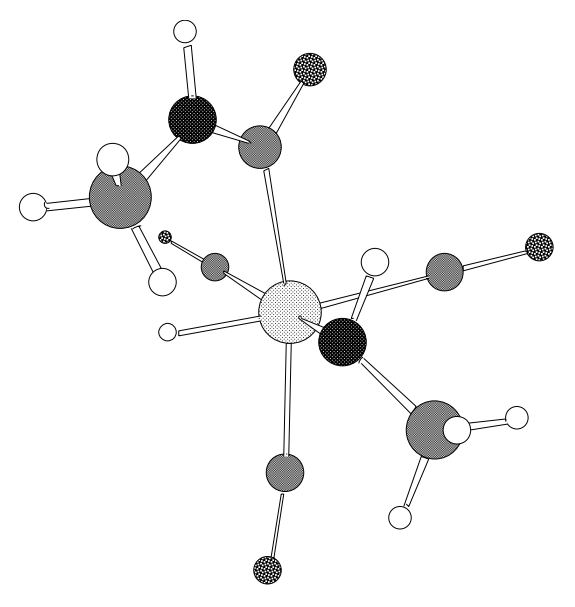

SCF Done: $\quad E(R B+H F-L Y P)=-789.473971555 \quad$ A.U.

\begin{tabular}{|c|c|c|c|c|c|}
\hline \multirow{2}{*}{$\begin{array}{l}\text { Center } \\
\text { Number }\end{array}$} & \multirow{2}{*}{$\begin{array}{l}\text { Atomic } \\
\text { Number }\end{array}$} & \multirow{2}{*}{$\begin{array}{l}\text { Atomic } \\
\text { Type }\end{array}$} & \multicolumn{3}{|c|}{ Coordinates (Angstroms) } \\
\hline & & & $\mathrm{X}$ & Y & $\mathrm{Z}$ \\
\hline 1 & 27 & 0 & 0.310923 & 0.236372 & -0.104118 \\
\hline
\end{tabular}




$\begin{array}{rrrrrr}2 & 7 & 0 & -2.558535 & -0.695860 & -0.140406 \\ 3 & 1 & 0 & -3.508347 & -0.454047 & 0.131210 \\ 4 & 6 & 0 & -2.432653 & -1.737089 & -1.158186 \\ 5 & 1 & 0 & -2.836681 & -1.408125 & -2.125310 \\ 6 & 1 & 0 & -2.989715 & -2.620237 & -0.829981 \\ 7 & 1 & 0 & -1.379140 & -2.006097 & -1.253897 \\ 8 & 6 & 0 & 0.689016 & 0.258282 & 1.685319 \\ 9 & 8 & 0 & 0.889323 & 0.179062 & 2.810729 \\ 10 & 6 & 0 & 1.800554 & -0.056864 & -1.194305 \\ 11 & 8 & 0 & 2.626251 & -0.304382 & -1.945494 \\ 12 & 6 & 0 & 0.318631 & 2.071762 & -0.362410 \\ 13 & 8 & 0 & 0.347126 & 3.197711 & -0.534543 \\ 14 & 6 & 0 & -1.722522 & 0.368355 & 0.044178 \\ 15 & 8 & 0 & -2.117074 & 1.422929 & 0.516967 \\ 16 & 7 & 0 & 0.239068 & -1.724145 & 0.180800 \\ 17 & 1 & 0 & -0.495784 & -1.853851 & 0.877689 \\ 18 & 1 & 0 & -0.636567 & 0.152299 & -1.291178 \\ 19 & 6 & 0 & 1.425885 & -2.440161 & 0.647094 \\ 20 & 1 & 0 & 2.206997 & -2.419720 & -0.121331 \\ 21 & 1 & 0 & 1.158176 & -3.495967 & 0.802000 \\ 22 & 1 & 0 & 1.883912 & -2.084532 & 1.587818 \\ -------------------------------------------------------------------------------------\end{array}$

\section{Cartesian coordinates of CP6b (B3LYP-631LAN)}
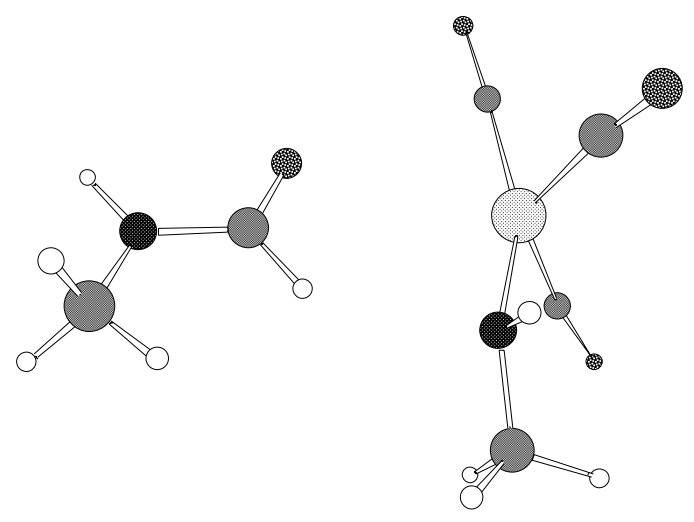

SCF Done: $\quad E(R B+H F-L Y P)=-789.540926360$

A.U. 


\begin{tabular}{cccccc}
$\begin{array}{l}\text { Center } \\
\text { Number }\end{array}$ & $\begin{array}{l}\text { Atomic } \\
\text { Number }\end{array}$ & $\begin{array}{l}\text { Atomic } \\
\text { Type }\end{array}$ & \multicolumn{4}{c}{ Coordinates (Angstroms) } \\
\hline----------------------------------------- \\
1 & 27 & 0 & -0.969412 & -0.035056 & 0.077326 \\
2 & 7 & 0 & 3.702713 & -0.544151 & -0.186951 \\
3 & 1 & 0 & 4.023499 & -1.422522 & -0.577870 \\
4 & 6 & 0 & 4.681207 & 0.440595 & 0.235462 \\
5 & 1 & 0 & 5.309220 & 0.767787 & -0.601819 \\
6 & 1 & 0 & 5.329534 & 0.047594 & 1.027631 \\
7 & 1 & 0 & 4.152899 & 1.313801 & 0.628089 \\
8 & 6 & 0 & -1.601015 & -0.405963 & 1.710011 \\
9 & 8 & 0 & -2.116509 & -0.573654 & 2.725878 \\
10 & 6 & 0 & -1.718456 & 0.705869 & -1.369496 \\
11 & 8 & 0 & -2.325519 & 1.194962 & -2.216138 \\
12 & 6 & 0 & -1.153657 & -1.725607 & -0.537014 \\
13 & 8 & 0 & -1.224660 & -2.800560 & -0.928607 \\
14 & 6 & 0 & 2.359373 & -0.383585 & -0.112360 \\
15 & 8 & 0 & 1.547712 & -1.228583 & -0.480864 \\
16 & 7 & 0 & -0.145955 & 1.521034 & 0.663065 \\
17 & 1 & 0 & -0.212122 & 1.668404 & 1.669108 \\
18 & 1 & 0 & 2.029571 & 0.580727 & 0.313369 \\
19 & 6 & 0 & -0.202459 & 2.837584 & 0.031551 \\
20 & 1 & 0 & -0.025643 & 2.747221 & -1.045102 \\
21 & 1 & 0 & 0.589162 & 3.484046 & 0.441288 \\
22 & 1 & 0 & -1.157453 & 3.370582 & 0.173643 \\
-------------------------------------------------------------------------------
\end{tabular}

\section{Cartesian coordinates of $\mathrm{CH}_{3} \mathrm{HNC}(=\mathrm{O}) \mathrm{NHCH}_{3}(\mathrm{B3LYP}-631 \mathrm{LAN})$}

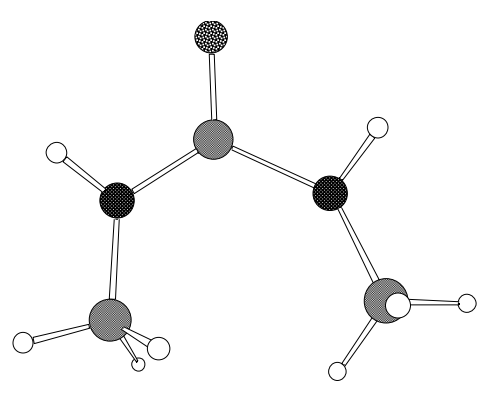


SCF Done: $\quad \mathrm{E}(\mathrm{RB}+\mathrm{HF}-\mathrm{LYP})=-303.870822634 \quad$ A.U.

\begin{tabular}{cccccc} 
Center & Atomic & Atomic & \multicolumn{3}{c}{ Coordinates (Angstroms) } \\
Number & Number & Type & $\mathrm{X}$ & $\mathrm{Y}$ & $\mathrm{Z}$ \\
\hline 1 & 7 & 0 & -1.112281 & 0.195319 & 0.364456 \\
2 & 1 & 0 & -1.775032 & 0.910302 & 0.633177 \\
3 & 6 & 0 & -1.680574 & -1.009541 & -0.232568 \\
4 & 1 & 0 & -1.143113 & -1.270787 & -1.148603 \\
5 & 1 & 0 & -2.727489 & -0.827185 & -0.495313 \\
6 & 1 & 0 & -1.650789 & -1.868390 & 0.450607 \\
7 & 6 & 0 & 0.082987 & 0.781247 & -0.010709 \\
8 & 8 & 0 & 0.247980 & 1.995872 & 0.027020 \\
9 & 7 & 0 & 1.087090 & -0.086219 & -0.447937 \\
10 & 1 & 0 & 1.899365 & 0.478891 & -0.669521 \\
11 & 6 & 0 & 1.430248 & -1.309560 & 0.280805 \\
12 & 1 & 0 & 0.625131 & -2.044328 & 0.223221 \\
13 & 1 & 0 & 1.657144 & -1.129795 & 1.341590 \\
14 & 1 & 0 & 2.311317 & -1.752250 & -0.192125 \\
-----125 & & & & &
\end{tabular}

\section{Cartesian coordinates of $\mathrm{HCo}(\mathrm{CO})_{3}$ (B3LYP-631LAN)}

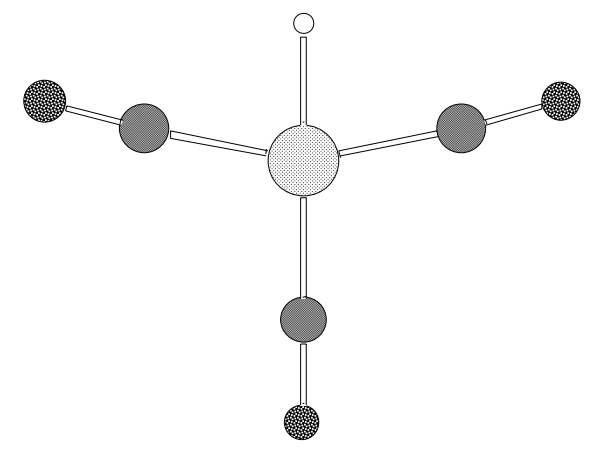

SCF Done: $\quad \mathrm{E}(\mathrm{RB}+\mathrm{HF}-\mathrm{LYP})=-485.671931731 \quad$ A.U.

\begin{tabular}{lllll} 
Center & Atomic & Atomic & \multicolumn{2}{l}{ Coordinates (Angstroms) } \\
Number & Number & Type & $X$ & $Y$
\end{tabular}




$\begin{array}{rccccc}1 & 27 & 0 & 0.003920 & -0.253696 & 0.000000 \\ 2 & 6 & 0 & 0.005692 & -0.610538 & 1.742090 \\ 3 & 8 & 0 & 0.005692 & -0.916784 & 2.847436 \\ 4 & 6 & 0 & -0.013760 & 1.531381 & 0.000000 \\ 5 & 8 & 0 & -0.025192 & 2.679389 & 0.000000 \\ 6 & 6 & 0 & 0.005692 & -0.610538 & -1.742090 \\ 7 & 8 & 0 & 0.005692 & -0.916784 & -2.847436 \\ 8 & 1 & 0 & 0.018859 & -1.778603 & 0.000000\end{array}$

\section{Cartesian coordinates of $\mathrm{NH}_{2} \mathrm{CH}_{3}$ (B3LYP-631LAN)}

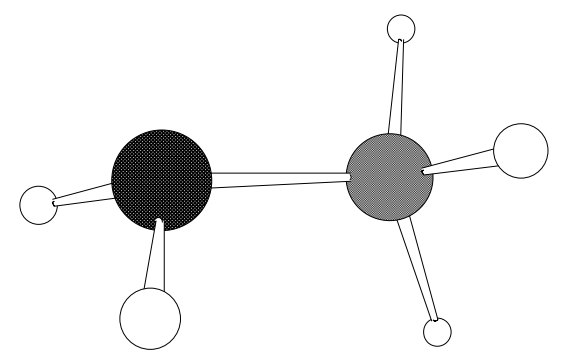

SCF Done: $\quad \mathrm{E}(\mathrm{RB}+\mathrm{HF}-\mathrm{LYP})=-95.8532048413 \quad$ A.U.

\begin{tabular}{cccccc} 
Center & Atomic & Atomic & \multicolumn{3}{c}{ Coordinates (Angstroms) } \\
Number & Number & Type & $X$ & Y & Z \\
-1 & 7 & 0 & -0.752337 & 0.000000 & -0.126122 \\
2 & 1 & 0 & -1.142762 & 0.812276 & 0.348683 \\
3 & 1 & 0 & -1.142731 & -0.812289 & 0.348686 \\
4 & 6 & 0 & 0.705767 & 0.000000 & 0.017592 \\
5 & 1 & 0 & 1.115529 & 0.881722 & -0.487222 \\
6 & 1 & 0 & 1.115539 & -0.881633 & -0.487369 \\
7 & 1 & 0 & 1.086180 & -0.000076 & 1.054524
\end{tabular}

\section{Cartesian coordinates of CO (B3LYP-631LAN)}




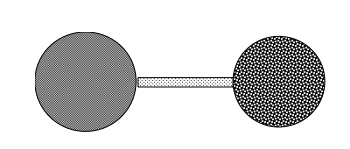

SCF Done: $\quad E(R B+H F-L Y P)=-113.309454336 \quad$ A.U.

\begin{tabular}{cccccc}
$\begin{array}{c}\text { Center } \\
\text { Number }\end{array}$ & Atomic & Atomic & \multicolumn{3}{c}{ Coordinates (Angstroms) } \\
Number & Type & $X$ & $Y$ & $Z$ \\
1 & 6 & 0 & 0.000000 & 0.000000 & -0.650253 \\
2 & 8 & 0 & 0.000000 & 0.000000 & 0.487690
\end{tabular}

\section{Optimized Geometries and energies of the Addition-Insertion} pathway:

Cartesian coordinates of TSa (B3LYP-631LAN)

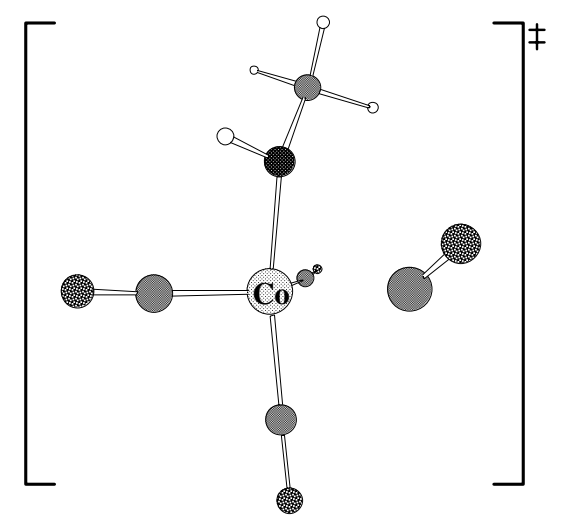

SCF Done: $\quad \mathrm{E}(\mathrm{RB}+\mathrm{HF}-\mathrm{LYP})=-693.645530904 \quad$ A.U.

\begin{tabular}{|c|c|c|c|c|c|}
\hline \multirow{2}{*}{$\begin{array}{l}\text { Center } \\
\text { Number }\end{array}$} & \multirow{2}{*}{$\begin{array}{l}\text { Atomic } \\
\text { Number }\end{array}$} & \multirow{2}{*}{$\begin{array}{l}\text { Atomic } \\
\text { Type }\end{array}$} & \multicolumn{3}{|c|}{ Coordinates (Angstroms) } \\
\hline & & & $\mathrm{X}$ & $\mathrm{Y}$ & $\mathrm{Z}$ \\
\hline 1 & 27 & 0 & 0.223317 & -0.113032 & 0.040229 \\
\hline 2 & 7 & 0 & -1.485046 & -0.187477 & 0.805567 \\
\hline
\end{tabular}




$\begin{array}{rrrrrr}3 & 1 & 0 & -1.472753 & -0.349136 & 1.810743 \\ 4 & 6 & 0 & -2.651719 & -0.876090 & 0.264610 \\ 5 & 1 & 0 & -3.551539 & -0.548452 & 0.806248 \\ 6 & 1 & 0 & -2.614578 & -1.976851 & 0.334779 \\ 7 & 1 & 0 & -2.793121 & -0.611116 & -0.788656 \\ 8 & 6 & 0 & 1.806682 & 0.394913 & -0.689165 \\ 9 & 8 & 0 & 2.801708 & 0.731306 & -1.148254 \\ 10 & 6 & 0 & 0.947934 & -0.571533 & 1.616137 \\ 11 & 8 & 0 & 1.385359 & -0.986596 & 2.594712 \\ 12 & 6 & 0 & -0.240741 & -1.109989 & -1.381227 \\ 13 & 8 & 0 & -0.486806 & -1.863645 & -2.212544 \\ 14 & 6 & 0 & -0.519766 & 2.265368 & -0.201035 \\ 15 & 8 & 0 & -1.357332 & 3.023155 & -0.051939\end{array}$

\section{Cartesian coordinates of CPD1 (B3LYP-631LAN)}

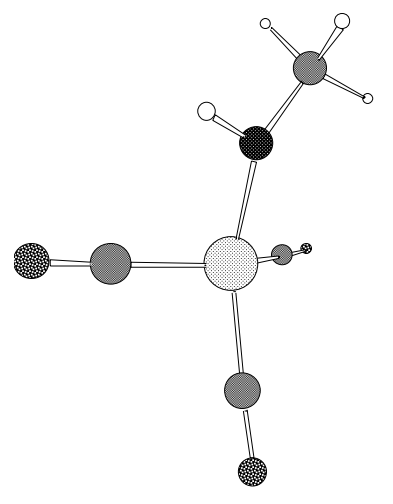

SCF Done: $\quad \mathrm{E}(\mathrm{RB}+\mathrm{HF}-\mathrm{LYP})=-580.337548649 \quad$ A.U.

\begin{tabular}{cccccc}
$\begin{array}{c}\text { Center } \\
\text { Number }\end{array}$ & Atomic & Atomic & \multicolumn{3}{c}{ Coordinates (Angstroms) } \\
- & Number & Type & X & Y & $Z$ \\
1 & 27 & 0 & 0.116892 & 0.040540 & -0.170085 \\
2 & 7 & 0 & -1.381873 & 0.942598 & -0.636765 \\
3 & 1 & 0 & -1.329092 & 1.958037 & -0.583869 \\
4 & 6 & 0 & -2.782857 & 0.555527 & -0.542956 \\
5 & 1 & 0 & -3.355407 & 1.046117 & -1.343981
\end{tabular}




$\begin{array}{rrrrrr}6 & 1 & 0 & -3.251848 & 0.838765 & 0.413362 \\ 7 & 1 & 0 & -2.890962 & -0.524424 & -0.673510 \\ 8 & 6 & 0 & 1.631685 & -0.868492 & -0.488581 \\ 9 & 8 & 0 & 2.589458 & -1.445471 & -0.753373 \\ 10 & 6 & 0 & 0.964983 & 1.497267 & 0.456528 \\ 11 & 8 & 0 & 1.465747 & 2.401926 & 0.958469 \\ 12 & 6 & 0 & -0.707111 & -1.360318 & 0.591815 \\ 13 & 8 & 0 & -1.217190 & -2.200851 & 1.187007\end{array}$

\section{Cartesian coordinates of CPD2 (B3LYP-631LAN)}

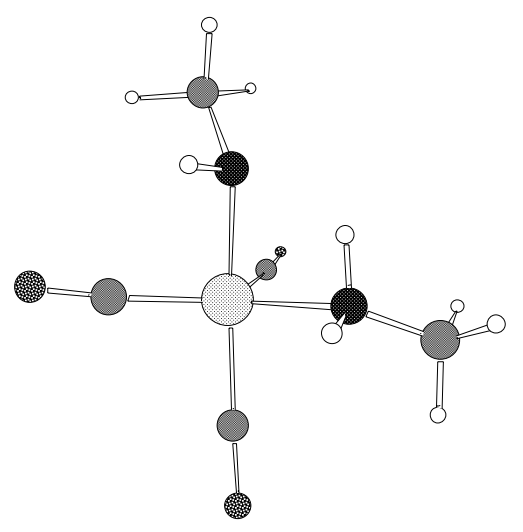

SCF Done: $\quad \mathrm{E}(\mathrm{RB}+\mathrm{HF}-\mathrm{LYP})=-676.205501321 \quad$ A.U.

\begin{tabular}{cccccc} 
Center & Atomic & Atomic & \multicolumn{3}{c}{ Coordinates (Angstroms) } \\
Number & Number & Type & $X$ & Y & $Z$ \\
-1 & 27 & 0 & -0.017053 & 0.215290 & -0.034104 \\
2 & 7 & 0 & -1.328285 & -0.979899 & -0.891323 \\
3 & 1 & 0 & -1.710916 & -0.475330 & -1.693147 \\
4 & 6 & 0 & -2.480061 & -1.491848 & -0.148140 \\
5 & 1 & 0 & -3.094253 & -2.123831 & -0.807695 \\
6 & 1 & 0 & -3.144751 & -0.718654 & 0.273899 \\
7 & 1 & 0 & -2.141664 & -2.122097 & 0.681664 \\
8 & 6 & 0 & 1.338781 & 1.310251 & 0.411210 \\
9 & 8 & 0 & 2.228042 & 1.998505 & 0.649787 \\
10 & 6 & 0 & -1.152083 & 1.509967 & -0.449930
\end{tabular}




\begin{tabular}{|c|c|c|c|c|c|}
\hline 11 & 8 & 0 & -1.976777 & 2.287871 & -0.671601 \\
\hline 12 & 6 & 0 & -0.118225 & -0.649859 & 1.572174 \\
\hline 13 & 8 & 0 & -0.263968 & -1.100894 & 2.620426 \\
\hline 14 & 7 & 0 & 1.296177 & -0.886903 & -1.336249 \\
\hline 15 & 1 & 0 & 0.505526 & -1.515422 & -1.532354 \\
\hline 16 & 1 & 0 & 1.505162 & -0.369898 & -2.188627 \\
\hline 17 & 6 & 0 & 2.490634 & -1.603390 & -0.859709 \\
\hline 18 & 1 & 0 & 2.914584 & -2.274219 & -1.618774 \\
\hline 19 & 1 & 0 & 3.260199 & -0.884993 & -0.561735 \\
\hline 20 & 1 & 0 & 2.218660 & -2.195351 & 0.018062 \\
\hline
\end{tabular}

\section{Cartesian coordinates of TSb (B3LYP-631LAN)}

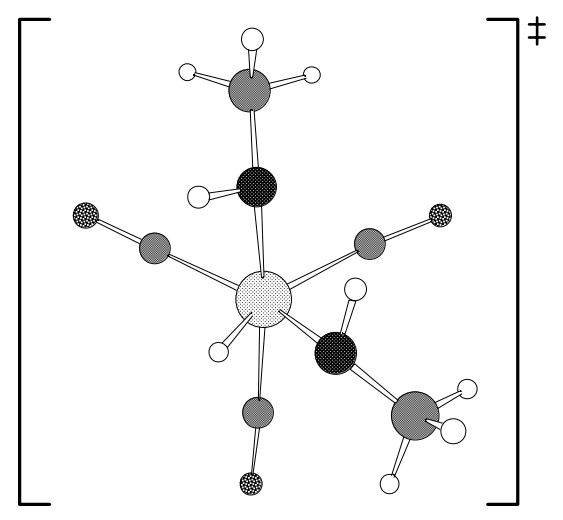

SCF Done: $\quad \mathrm{E}(\mathrm{RB}+\mathrm{HF}-\mathrm{LYP})=-676.109185756 \quad$ A.U.

\begin{tabular}{cccccc} 
Center & Atomic & Atomic & \multicolumn{3}{c}{ Coordinates (Angstroms) } \\
Number & Number & Type & X & Y & $Z$ \\
-1 & 27 & 0 & 0.023632 & 0.226453 & -0.145924 \\
2 & 7 & 0 & -1.385295 & -0.940206 & -0.926263 \\
3 & 1 & 0 & -1.654157 & -0.506706 & -1.809917 \\
4 & 6 & 0 & -2.604528 & -1.273341 & -0.192360 \\
5 & 1 & 0 & -3.186668 & -1.992056 & -0.788101 \\
6 & 1 & 0 & -3.283934 & -0.437230 & 0.053224 \\
7 & 1 & 0 & -2.350303 & -1.773660 & 0.749800 \\
8 & 6 & 0 & 1.361560 & 1.392482 & 0.268794
\end{tabular}




$\begin{array}{rrrrrr}9 & 8 & 0 & 2.170060 & 2.167780 & 0.498849 \\ 10 & 6 & 0 & -1.365947 & 1.377490 & 0.044406 \\ 11 & 8 & 0 & -2.285541 & 2.062603 & 0.044458 \\ 12 & 6 & 0 & 0.105411 & -0.899977 & 1.400778 \\ 13 & 8 & 0 & 0.262982 & -1.726220 & 2.172682 \\ 14 & 7 & 0 & 1.210040 & -0.876621 & -1.276796 \\ 15 & 1 & 0 & 0.587552 & -1.678487 & -1.358110 \\ 16 & 1 & 0 & 0.359502 & 0.576392 & -1.536046 \\ 17 & 6 & 0 & 2.534350 & -1.213707 & -0.793044 \\ 18 & 1 & 0 & 2.994978 & -1.936852 & -1.482101 \\ 19 & 1 & 0 & 3.179575 & -0.325951 & -0.790029 \\ 20 & 1 & 0 & 2.577086 & -1.652876 & 0.223281 \\ ------------------~\end{array}$

\section{Cartesian coordinates of CPD3 (B3LYP-631LAN)}

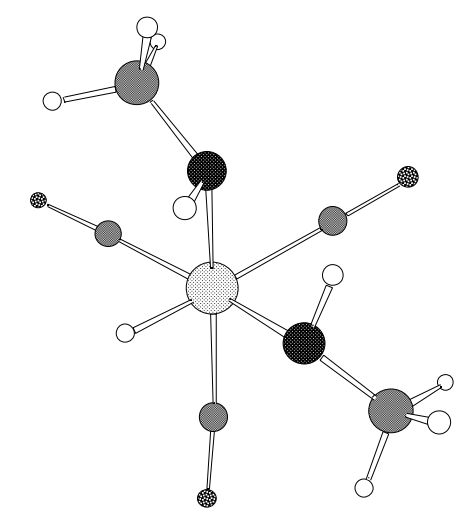

SCF Done: $\quad \mathrm{E}(\mathrm{RB}+\mathrm{HF}-\mathrm{LYP})=-676.117538930 \quad$ A.U.

\begin{tabular}{cccccc} 
Center & Atomic & Atomic & \multicolumn{3}{c}{ Coordinates (Angstroms) } \\
Number & Number & Type & X & Y & Z \\
\hline 1 & 27 & 0 & -0.022055 & 0.209521 & -0.159612 \\
2 & 7 & 0 & -1.215089 & -1.236884 & -0.792137 \\
3 & 1 & 0 & -0.963375 & -1.256087 & -1.781115 \\
4 & 6 & 0 & -2.668057 & -1.134294 & -0.702283 \\
5 & 1 & 0 & -3.108678 & -2.052012 & -1.120717 \\
6 & 1 & 0 & -3.134482 & -0.283854 & -1.231178
\end{tabular}




\begin{tabular}{|c|c|c|c|c|c|}
\hline 7 & 1 & 0 & -2.982107 & -1.085811 & 0.348192 \\
\hline 8 & 6 & 0 & 1.262293 & 1.507760 & 0.016642 \\
\hline 9 & 8 & 0 & 2.037220 & 2.346431 & 0.013005 \\
\hline 10 & 6 & 0 & -1.439751 & 1.265524 & 0.296853 \\
\hline 11 & 8 & 0 & -2.345947 & 1.933968 & 0.487903 \\
\hline 12 & 6 & 0 & 0.179604 & -0.897919 & 1.371695 \\
\hline 13 & 8 & 0 & 0.308627 & -1.713421 & 2.158189 \\
\hline 14 & 7 & 0 & 1.330302 & -0.956540 & -1.027079 \\
\hline 15 & 1 & 0 & 0.867181 & -1.857074 & -0.889705 \\
\hline 16 & 1 & 0 & -0.196230 & 0.821341 & -1.491233 \\
\hline 17 & 6 & 0 & 2.680458 & -1.052108 & -0.480312 \\
\hline 18 & 1 & 0 & 3.210322 & -1.860347 & -1.006204 \\
\hline 19 & 1 & 0 & 3.242485 & -0.130897 & -0.675406 \\
\hline 20 & 1 & 0 & 2.767410 & -1.267973 & 0.603052 \\
\hline
\end{tabular}

\section{Cartesian coordinates of TSc (B3LYP-631LAN)}

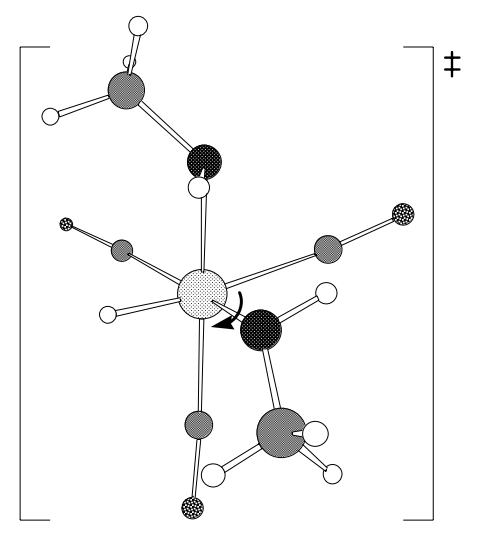

SCF Done: $\quad \mathrm{E}(\mathrm{RB}+\mathrm{HF}-\mathrm{LYP})=-676.115945543 \quad$ A.U.

\begin{tabular}{cccccc}
$\begin{array}{l}\text { Center } \\
\text { Number }\end{array}$ & Atomic & Atomic & \multicolumn{3}{c}{ Coordinates (Angstroms) } \\
& Number & Type & $X$ & Y & $Z$ \\
\hline 1 & 27 & 0 & 0.004710 & 0.137847 & -0.096929 \\
2 & 7 & 0 & -1.302426 & -1.275640 & -0.517062 \\
3 & 1 & 0 & -0.742217 & -1.723366 & -1.245319 \\
4 & 6 & 0 & -2.616679 & -0.962965 & -1.065363
\end{tabular}




\begin{tabular}{|c|c|c|c|c|c|}
\hline 5 & 1 & 0 & -3.115644 & -1.907792 & -1.329805 \\
\hline 6 & 1 & 0 & -2.620113 & -0.328282 & -1.969549 \\
\hline 7 & 1 & 0 & -3.248786 & -0.477020 & -0.311575 \\
\hline 8 & 6 & 0 & 1.395319 & 1.327587 & 0.100313 \\
\hline 9 & 8 & 0 & 2.261358 & 2.071742 & 0.124168 \\
\hline 10 & 6 & 0 & -1.318316 & 1.378776 & 0.057772 \\
\hline 11 & 8 & 0 & -2.138120 & 2.174275 & 0.056759 \\
\hline 12 & 6 & 0 & -0.096098 & -0.709112 & 1.613538 \\
\hline 13 & 8 & 0 & -0.204331 & -1.360710 & 2.543198 \\
\hline 14 & 7 & 0 & 1.247402 & -1.278987 & -0.748029 \\
\hline 15 & 1 & 0 & 1.127761 & -2.026308 & -0.061231 \\
\hline 16 & 1 & 0 & -0.007710 & 0.543818 & -1.515185 \\
\hline 17 & 6 & 0 & 2.680708 & -1.039289 & -0.880256 \\
\hline 18 & 1 & 0 & 3.159573 & -1.967476 & -1.227563 \\
\hline 19 & 1 & 0 & 2.861808 & -0.278840 & -1.649095 \\
\hline 20 & 1 & 0 & 3.222466 & -0.726662 & 0.033009 \\
\hline
\end{tabular}

\section{Cartesian coordinates of CPD4 (B3LYP-631LAN)}

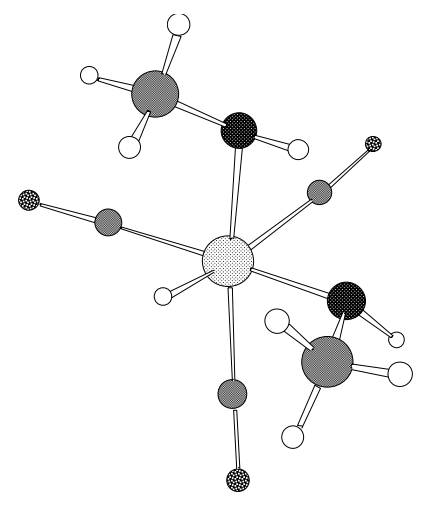

SCF Done: $\quad \mathrm{E}(\mathrm{RB}+\mathrm{HF}-\mathrm{LYP})=-676.122083095 \quad$ A.U.

\begin{tabular}{cccccc} 
Center & Atomic & Atomic & \multicolumn{3}{c}{ Coordinates (Angstroms) } \\
Number & Number & Type & $X$ & Y & $Z$ \\
\hline 1 & 27 & 0 & -0.018883 & -0.164122 & -0.043349 \\
2 & 7 & 0 & 1.263805 & 1.301796 & 0.170814
\end{tabular}




\begin{tabular}{|c|c|c|c|c|c|}
\hline 3 & 1 & 0 & 0.567056 & 2.023291 & 0.366213 \\
\hline 4 & 6 & 0 & 2.072819 & 1.726610 & -0.965749 \\
\hline 5 & 1 & 0 & 2.645791 & 2.620745 & -0.675256 \\
\hline 6 & 1 & 0 & 1.507431 & 1.976819 & -1.880830 \\
\hline 7 & 1 & 0 & 2.807149 & 0.956415 & -1.232017 \\
\hline 8 & 6 & 0 & -1.485788 & -1.256918 & -0.310868 \\
\hline 9 & 8 & 0 & -2.478127 & -1.768167 & -0.565886 \\
\hline 10 & 6 & 0 & 1.307851 & -1.227445 & -0.708838 \\
\hline 11 & 8 & 0 & 2.119110 & -1.858633 & -1.205646 \\
\hline 12 & 6 & 0 & 0.381585 & -0.336434 & 1.827062 \\
\hline 13 & 8 & 0 & 0.696228 & -0.285455 & 2.919830 \\
\hline 14 & 7 & 0 & -1.400787 & 1.175563 & 0.395344 \\
\hline 15 & 1 & 0 & -2.066012 & 0.757459 & 1.049529 \\
\hline 16 & 1 & 0 & -0.192166 & 0.214436 & -1.454626 \\
\hline 17 & 6 & 0 & -2.171376 & 1.760308 & -0.702953 \\
\hline 18 & 1 & 0 & -2.855447 & 2.520115 & -0.295180 \\
\hline 19 & 1 & 0 & -1.490430 & 2.266694 & -1.394784 \\
\hline 20 & 1 & 0 & -2.782906 & 1.055138 & -1.294041 \\
\hline
\end{tabular}

\section{Cartesian coordinates of TSd (B3LYP-631LAN)}

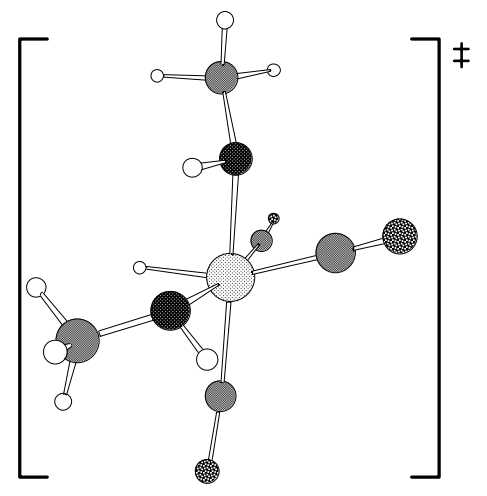

SCF Done: $\quad \mathrm{E}(\mathrm{RB}+\mathrm{HF}-\mathrm{LYP})=-676.120230551 \quad$ A.U.

\begin{tabular}{lllll} 
Center & Atomic & Atomic & \multicolumn{2}{c}{ Coordinates (Angstroms) } \\
Number & Number & Type & $X$ & $Y$ \\
\end{tabular}




\begin{tabular}{rrrrrr}
1 & 27 & 0 & 0.074289 & 0.173822 & -0.039211 \\
2 & 7 & 0 & -1.290401 & -1.188503 & -0.321569 \\
3 & 1 & 0 & -0.688368 & -1.960792 & -0.601170 \\
4 & 6 & 0 & -2.441765 & -0.990317 & -1.180718 \\
5 & 1 & 0 & -3.039594 & -1.912487 & -1.216635 \\
6 & 1 & 0 & -2.186790 & -0.709672 & -2.217213 \\
7 & 1 & 0 & -3.091772 & -0.205096 & -0.774331 \\
8 & 6 & 0 & 1.604178 & 1.079484 & 0.358035 \\
9 & 8 & 0 & 2.627510 & 1.560869 & 0.537835 \\
10 & 6 & 0 & -1.038402 & 1.572575 & -0.358262 \\
11 & 8 & 0 & -1.682700 & 2.468636 & -0.654686 \\
12 & 6 & 0 & -0.697737 & -0.503456 & 1.539081 \\
13 & 8 & 0 & -1.172198 & -0.936605 & 2.493746 \\
14 & 7 & 0 & 1.343657 & -1.340433 & -0.103030 \\
15 & 1 & 0 & 1.853035 & -1.372757 & 0.782037 \\
16 & 1 & 0 & 0.376323 & 0.402503 & -1.483193 \\
17 & 6 & 0 & 2.320101 & -1.419420 & -1.190483 \\
18 & 1 & 0 & 2.891203 & -2.354145 & -1.084009 \\
19 & 1 & 0 & 1.794021 & -1.458364 & -2.149319 \\
20 & 1 & 0 & 3.054211 & -0.596235 & -1.246337 \\
\hline----------------------------------------------------------------------19
\end{tabular}

\section{Cartesian coordinates of CPD5 (B3LYP-631LAN)}

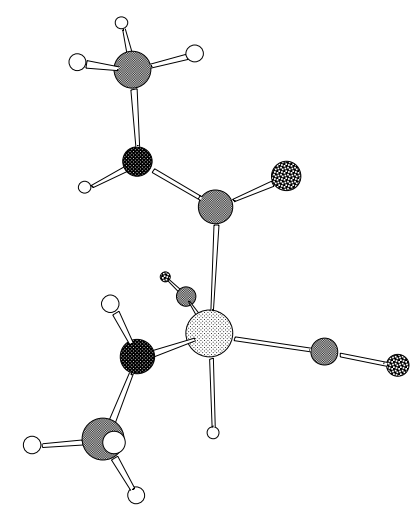

SCF Done: $\quad \mathrm{E}(\mathrm{RB}+\mathrm{HF}-\mathrm{LYP})=-676.154852466 \quad$ A.U.

Center Atomic Atomic Coordinates (Angstroms)




\begin{tabular}{|c|c|c|c|c|c|}
\hline Number & Number & Type & $X$ & $\mathrm{Y}$ & $\mathrm{Z}$ \\
\hline 1 & 27 & 0 & 0.713786 & 0.122167 & 0.119271 \\
\hline 2 & 7 & 0 & -2.180991 & 0.189552 & 0.211681 \\
\hline 3 & 1 & 0 & -1.974043 & 0.555921 & 1.129640 \\
\hline 4 & 6 & 0 & -3.578180 & 0.001529 & -0.164629 \\
\hline 5 & 1 & 0 & -4.123065 & 0.951115 & -0.122835 \\
\hline 6 & 1 & 0 & -4.078783 & -0.723727 & 0.489130 \\
\hline 7 & 1 & 0 & -3.591232 & -0.378232 & -1.186847 \\
\hline 8 & 6 & 0 & 1.614319 & -1.079382 & -0.846423 \\
\hline 9 & 8 & 0 & 2.223401 & -1.793432 & -1.501315 \\
\hline 10 & 6 & 0 & 0.474860 & -0.952645 & 1.511228 \\
\hline 11 & 8 & 0 & 0.351040 & -1.580936 & 2.464819 \\
\hline 12 & 6 & 0 & -1.137297 & -0.331650 & -0.508022 \\
\hline 13 & 8 & 0 & -1.294149 & -0.933635 & -1.558216 \\
\hline 14 & 7 & 0 & 0.347316 & 1.838651 & -0.062467 \\
\hline 15 & 1 & 0 & -0.628491 & 2.104183 & -0.202242 \\
\hline 16 & 1 & 0 & 2.187344 & 0.455112 & 0.458593 \\
\hline 17 & 6 & 0 & 1.182653 & 3.024541 & -0.008029 \\
\hline 18 & 1 & 0 & 0.937935 & 3.638129 & 0.872952 \\
\hline 19 & 1 & 0 & 2.233963 & 2.740075 & 0.047224 \\
\hline 20 & 1 & 0 & 1.019423 & 3.651169 & -0.897510 \\
\hline
\end{tabular}

\section{Cartesian coordinates of TSe (B3LYP-631LAN)}

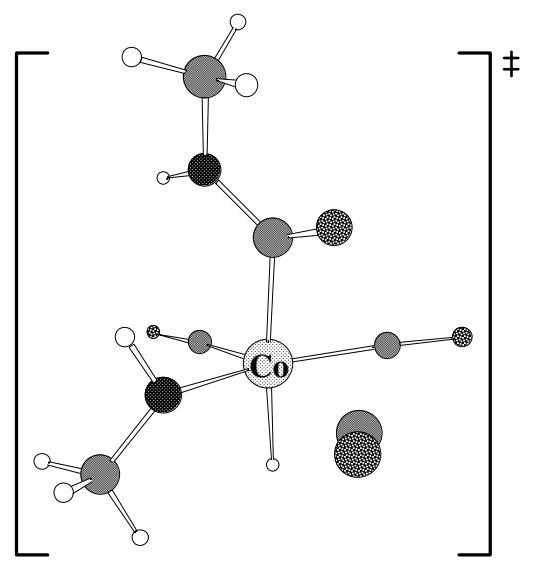




\begin{tabular}{|c|c|c|c|c|c|}
\hline \multirow{2}{*}{$\begin{array}{l}\text { Center } \\
\text { Number }\end{array}$} & \multirow{2}{*}{$\begin{array}{l}\text { Atomic } \\
\text { Number }\end{array}$} & \multirow{2}{*}{$\begin{array}{l}\text { Atomic } \\
\text { Type }\end{array}$} & \multicolumn{3}{|c|}{ Coordinates (Angstroms) } \\
\hline & & & $\mathrm{X}$ & $\mathrm{Y}$ & $\mathrm{Z}$ \\
\hline 1 & 27 & 0 & 0.494564 & 0.322538 & 0.284119 \\
\hline 2 & 7 & 0 & -2.309429 & 0.022191 & -0.525386 \\
\hline 3 & 1 & 0 & -2.308710 & 1.029194 & -0.458434 \\
\hline 4 & 6 & 0 & -3.569386 & -0.647005 & -0.833639 \\
\hline 5 & 1 & 0 & -4.282840 & -0.581162 & -0.001967 \\
\hline 6 & 1 & 0 & -3.349587 & -1.698174 & -1.022363 \\
\hline 7 & 1 & 0 & -4.022844 & -0.203989 & -1.726228 \\
\hline 8 & 6 & 0 & 0.492697 & -0.243811 & 2.036801 \\
\hline 9 & 8 & 0 & 0.536169 & -0.581430 & 3.126707 \\
\hline 10 & 6 & 0 & 0.012891 & 1.957625 & 0.531961 \\
\hline 11 & 8 & 0 & -0.255822 & 3.069130 & 0.657010 \\
\hline 12 & 6 & 0 & -1.207434 & -0.644948 & -0.064992 \\
\hline 13 & 8 & 0 & -1.178100 & -1.866274 & 0.031236 \\
\hline 14 & 7 & 0 & 0.727119 & 0.534133 & -1.520622 \\
\hline 15 & 1 & 0 & -0.146993 & 0.532458 & -2.045955 \\
\hline 16 & 1 & 0 & 1.892311 & 0.938325 & 0.600396 \\
\hline 17 & 6 & 0 & 1.684103 & 1.419045 & -2.168391 \\
\hline 18 & 1 & 0 & 1.880412 & 1.048161 & -3.186065 \\
\hline 19 & 1 & 0 & 1.342499 & 2.463345 & -2.265090 \\
\hline 20 & 1 & 0 & 2.626052 & 1.414433 & -1.615851 \\
\hline 21 & 6 & 0 & 1.728818 & -1.960805 & -0.209765 \\
\hline 22 & 8 & 0 & 2.053067 & -2.724676 & -0.987384 \\
\hline
\end{tabular}

\section{Cartesian coordinates of CPD6 (B3LYP-631LAN)}




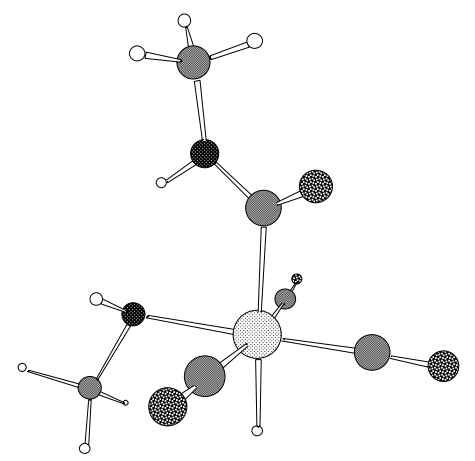

SCF Done: $\quad \mathrm{E}(\mathrm{RB}+\mathrm{HF}-\mathrm{LYP})=-789.488254381 \quad$ A.U.

\begin{tabular}{|c|c|c|c|c|c|}
\hline \multirow{2}{*}{$\begin{array}{l}\text { Center } \\
\text { Number }\end{array}$} & \multirow{2}{*}{$\begin{array}{l}\text { Atomic } \\
\text { Number }\end{array}$} & \multirow{2}{*}{$\begin{array}{l}\text { Atomic } \\
\text { Type }\end{array}$} & \multicolumn{3}{|c|}{ Coordinates (Angstroms) } \\
\hline & & & $\mathrm{X}$ & $\mathrm{Y}$ & $\mathrm{Z}$ \\
\hline 1 & 27 & 0 & -0.639856 & 0.121596 & 0.136034 \\
\hline 2 & 7 & 0 & 2.126928 & -0.540666 & -0.188026 \\
\hline 3 & 1 & 0 & 1.647361 & -1.427493 & -0.043716 \\
\hline 4 & 6 & 0 & 3.547497 & -0.495826 & -0.506188 \\
\hline 5 & 1 & 0 & 4.106875 & -1.121422 & 0.196607 \\
\hline 6 & 1 & 0 & 3.882956 & 0.538839 & -0.418138 \\
\hline 7 & 1 & 0 & 3.750403 & -0.844942 & -1.527405 \\
\hline 8 & 6 & 0 & -0.908154 & 1.876690 & 0.608486 \\
\hline 9 & 8 & 0 & -1.115504 & 2.958195 & 0.898621 \\
\hline 10 & 6 & 0 & -0.234284 & -0.466073 & 1.803734 \\
\hline 11 & 8 & 0 & -0.017914 & -0.924954 & 2.827326 \\
\hline 12 & 6 & 0 & 1.324236 & 0.544287 & -0.275894 \\
\hline 13 & 8 & 0 & 1.701105 & 1.675357 & -0.538252 \\
\hline 14 & 7 & 0 & -0.332930 & -1.787854 & -0.346816 \\
\hline 15 & 1 & 0 & -0.033487 & -1.797297 & -1.323099 \\
\hline 16 & 1 & 0 & -2.135387 & -0.131534 & 0.468145 \\
\hline 17 & 6 & 0 & -1.485433 & -2.681994 & -0.223867 \\
\hline 18 & 1 & 0 & -1.182393 & -3.693575 & -0.534549 \\
\hline 19 & 1 & 0 & -1.801806 & -2.735903 & 0.822414 \\
\hline 20 & 1 & 0 & -2.366077 & -2.395587 & -0.819323 \\
\hline 21 & 6 & 0 & -1.151114 & 0.284779 & -1.574957 \\
\hline 22 & 8 & 0 & -1.531040 & 0.323187 & -2.654924 \\
\hline
\end{tabular}




\section{Cartesian coordinates of TSf (B3LYP-631LAN)}

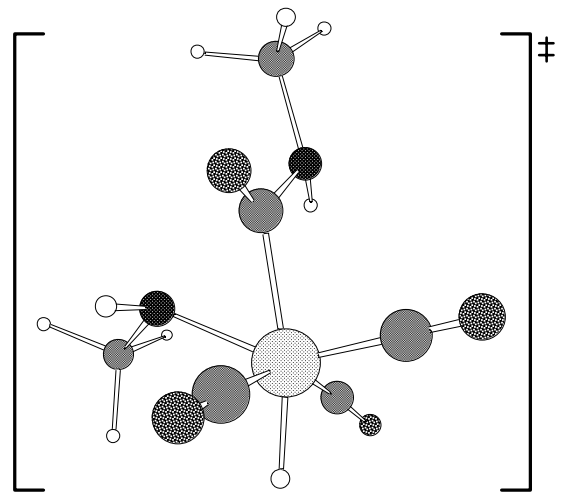

SCF Done: $\quad \mathrm{E}(\mathrm{RB}+\mathrm{HF}-\mathrm{LYP})=-789.463484977 \quad$ A.U.

\begin{tabular}{|c|c|c|c|c|c|}
\hline \multirow{2}{*}{$\begin{array}{l}\text { Center } \\
\text { Number }\end{array}$} & \multirow{2}{*}{$\begin{array}{l}\text { Atomic } \\
\text { Number }\end{array}$} & \multirow{2}{*}{$\begin{array}{l}\text { Atomic } \\
\text { Type }\end{array}$} & \multicolumn{3}{|c|}{ Coordinates (Angstroms) } \\
\hline & & & $X$ & $\mathrm{Y}$ & $\mathrm{Z}$ \\
\hline 1 & 27 & 0 & 0.732021 & 0.176297 & 0.133752 \\
\hline 2 & 7 & 0 & -2.083519 & -0.469968 & 0.442058 \\
\hline 3 & 1 & 0 & -2.055891 & 0.460189 & 0.836588 \\
\hline 4 & 6 & 0 & -3.409637 & -0.915219 & 0.006171 \\
\hline 5 & 1 & 0 & -4.120112 & -0.742117 & 0.818880 \\
\hline 6 & 1 & 0 & -3.367260 & -1.984074 & -0.207690 \\
\hline 7 & 1 & 0 & -3.753680 & -0.390416 & -0.896326 \\
\hline 8 & 6 & 0 & 0.975447 & -1.118514 & 1.383591 \\
\hline 9 & 8 & 0 & 1.136705 & -1.935851 & 2.169801 \\
\hline 10 & 6 & 0 & 0.311940 & 1.465044 & 1.302276 \\
\hline 11 & 8 & 0 & 0.117716 & 2.301549 & 2.065964 \\
\hline 12 & 6 & 0 & -1.014715 & -0.772280 & -0.373840 \\
\hline 13 & 8 & 0 & -0.972228 & -1.707549 & -1.141826 \\
\hline 14 & 7 & 0 & -0.421128 & 0.891400 & -1.344314 \\
\hline 15 & 1 & 0 & -0.102836 & 0.514677 & -2.231926 \\
\hline 16 & 1 & 0 & 1.955080 & 1.157089 & 0.257627 \\
\hline 17 & 6 & 0 & -0.615389 & 2.330460 & -1.404384 \\
\hline 18 & 1 & 0 & -1.159086 & 2.594324 & -2.322460 \\
\hline 19 & 1 & 0 & -1.230963 & 2.657984 & -0.555704 \\
\hline 20 & 1 & 0 & 0.317880 & 2.918576 & -1.376831 \\
\hline
\end{tabular}




$\begin{array}{llllll}21 & 6 & 0 & 1.834797 & -0.508042 & -1.105977 \\ 22 & 8 & 0 & 2.566578 & -0.881268 & -1.902025\end{array}$

\section{Cartesian coordinates of CPD7 (B3LYP-631LAN)}

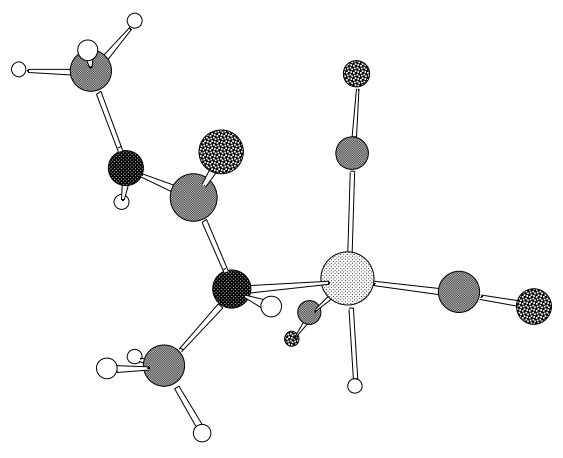

SCF Done: $\quad \mathrm{E}(\mathrm{RB}+\mathrm{HF}-\mathrm{LYP})=-789.560383202 \quad$ A.U.

\begin{tabular}{cccccc} 
Center & Atomic & Atomic & \multicolumn{3}{c}{ Coordinates (Angstroms) } \\
Number & Number & Type & X & Y & $Z$ \\
\hline 1 & 27 & 0 & 1.099175 & 0.076667 & -0.035598 \\
2 & 7 & 0 & -2.514035 & 0.466528 & 0.135913 \\
3 & 1 & 0 & -2.042700 & 1.334659 & 0.349306 \\
4 & 6 & 0 & -3.578105 & 0.040052 & 1.035230 \\
5 & 1 & 0 & -3.186775 & -0.300450 & 2.002454 \\
6 & 1 & 0 & -4.111232 & -0.788322 & 0.567723 \\
7 & 1 & 0 & -4.269156 & 0.871406 & 1.198231 \\
8 & 6 & 0 & 0.422637 & -0.659069 & 1.462376 \\
9 & 8 & 0 & 0.029411 & -1.129389 & 2.434024 \\
10 & 6 & 0 & 1.445367 & 1.690795 & 0.594378 \\
11 & 8 & 0 & 1.774355 & 2.729389 & 0.975751 \\
12 & 6 & 0 & -1.843102 & -0.449618 & -0.619776 \\
13 & 8 & 0 & -2.147338 & -1.626430 & -0.718923 \\
14 & 7 & 0 & -0.700933 & 0.075223 & -1.325216 \\
15 & 1 & 0 & -0.437755 & -0.664303 & -1.972917 \\
16 & 1 & 0 & 1.831880 & 0.688113 & -1.203670 \\
17 & 6 & 0 & -0.884428 & 1.349746 & -2.059829 \\
& & & & &
\end{tabular}




$\begin{array}{rlrrrr}18 & 1 & 0 & -1.794398 & 1.340975 & -2.671953 \\ 19 & 1 & 0 & -0.927451 & 2.188636 & -1.362081 \\ 20 & 1 & 0 & -0.012140 & 1.499409 & -2.697416 \\ 21 & 6 & 0 & 2.198966 & -1.241458 & -0.475730 \\ 22 & 8 & 0 & 2.994667 & -2.025454 & -0.758765\end{array}$

\section{Cartesian coordinates of $\mathrm{CH}_{3} \mathrm{HNC}(=\mathrm{O}) \mathrm{NHCH}_{3}(\mathrm{B3LYP}-631 \mathrm{LAN})$}

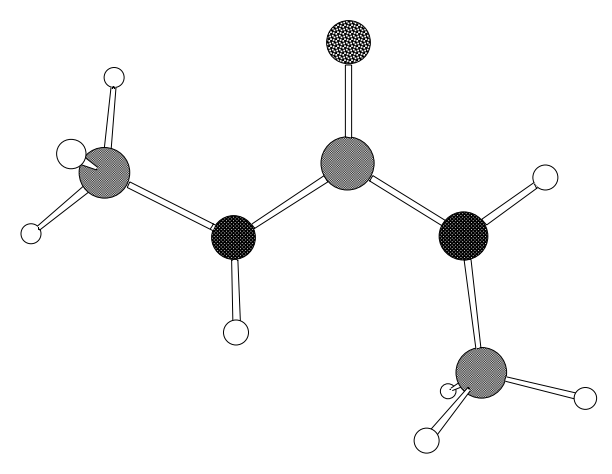

SCF Done: $\quad \mathrm{E}(\mathrm{RB}+\mathrm{HF}-\mathrm{LYP})=-303.877299541 \quad$ A.U.

\begin{tabular}{rrrrrr} 
Center & Atomic & Atomic & \multicolumn{3}{c}{ Coordinates (Angstroms) } \\
Number & Number & Type & X & Y & Z \\
\hline 1 & 7 & 0 & 0.710636 & -0.685726 & 0.033705 \\
2 & 1 & 0 & 0.244601 & -1.495915 & -0.350490 \\
3 & 6 & 0 & 2.164323 & -0.693752 & -0.007958 \\
4 & 1 & 0 & 2.533624 & -1.631897 & 0.417056 \\
5 & 1 & 0 & 2.527546 & 0.141853 & 0.591136 \\
6 & 1 & 0 & 2.562589 & -0.581258 & -1.026335 \\
7 & 6 & 0 & 0.040577 & 0.521368 & -0.021529 \\
8 & 8 & 0 & 0.603737 & 1.606149 & 0.082380 \\
9 & 7 & 0 & -1.332505 & 0.426610 & -0.196326 \\
10 & 1 & 0 & -1.774644 & 1.322461 & -0.043270 \\
11 & 6 & 0 & -2.123566 & -0.757301 & 0.097010 \\
12 & 1 & 0 & -1.953344 & -1.547072 & -0.647205 \\
13 & 1 & 0 & -1.923265 & -1.173520 & 1.094678 \\
14 & 1 & 0 & -3.181927 & -0.491920 & 0.038591
\end{tabular}


